\title{
SOLVING THEMATIC DIVERGENCES IN MACHINE TRANSLATION
}

\author{
Bonnie Dorr* \\ M.I.T. Artificial Intelligence Laboratory \\ 545 Technology Square, Room 810 \\ Cambridge, MA 02139, USA \\ internet: bonnie@reagan.ai.mit.edu
}

\begin{abstract}
Though most trenslation systems have some mechanism for translating certain types of divergent predicate-argument structures, they do not provide a general procedure that takes advantage of the relationship between lexical-semantic structure and syntactic structure. A divergent predicate-argument structure is one in which the predicate (e.g., the main verb) or its arguments (e.g., the subject and object) do not have the same syntactic ordering properties for both the source and target language. To account for such ordering differences, a machine translator must consider language-specific syntactic idiosyncrasies that distinguish a target language from a source language, while making use of lexical-semantic uniformities that tie the two languages together. This paper describes the mechanisms used by the UNITRAN machine translation system for mapping an underlying lexicalconceptual structure to a syntactic structure (and vice versa), and it shows how these mechanisms coupled with a set of general linking routines solve the problem of thematic divergence in machine translation.
\end{abstract}

\section{INTRODUCTION}

There are a number of different divergence types that arise during the translation of a source language to a target language. Figure 1 shows some of these divergences with respect to Spanish, English, and German. ${ }^{1}$

We will look at each of these traditionally difficult divergence types in turn. The first divergence type is a structural divergence in that the verbal object is realized as a noun phrase (John) in English and as a prepositional phrase (a Juan) in Spanish. The second diver-

*This paper describes research done at the Artificial Intelligence Laboratory of the Massachusetts Institute of Technology. Support for this research has been provided by NSF Grant DCR-85552543 under a Presidential Young Investigator's Award to Professor Robert C. Berwick. Useful guidance and commentary during this research were provided by Bob Berwick, Noam Chomsky, Bruce Dawson, Ken Hale, Mike Kashket, Jeff Siskind, and Patrick Winston. The author is also indebted to three anonymous reviewers for their aid in reshaping this paper into its current form.

${ }^{1}$ Many sentences may fit into these divergence classes, not just the ones listed here. Also, a single sentence may exhibit any or all of these divergences.

\begin{tabular}{|l|l|}
\hline $\begin{array}{c}\text { Divergence } \\
\text { Type }\end{array}$ & \multicolumn{1}{c|}{$\begin{array}{c}\text { Tnanslation } \\
\text { Example }\end{array}$} \\
\hline Structural & $\begin{array}{l}\text { I saw John } \\
\text { Vi a Juan } \\
\text { (I saw to John) }\end{array}$ \\
\hline Conflational & $\begin{array}{l}\text { I like Mary } \\
\text { I } \\
\text { Ich habe Marie gern } \\
\text { (I have Mary likingly) }\end{array}$ \\
\hline Lexical & $\begin{array}{l}\text { I stabbed John } \\
\text { I } \\
\text { Yo le di punaladas a Juan } \\
\text { (I gave knife-wounds to John) }\end{array}$ \\
\hline Categorial & $\begin{array}{l}\text { I am hungry } \\
\mathbb{1}\end{array}$ \\
$\begin{array}{l}\text { Ich habe Hunger } \\
\text { (I have hunger) }\end{array}$ \\
\hline Thematic & $\begin{array}{l}\text { I like Mary } \\
\text { Maria me gusta a mí } \\
\text { (Mary pleases me) }\end{array}$ \\
\hline
\end{tabular}

Figure 1: Divergence Types in Machine Translation

gence is conflational. Conflation is the incorporation of necessary participants (or arguments) of a given action. Here, English uses the single word like for the two German words haben (have) and gern (likingly); this is because the manner argument (i.e., the likingly portion of the lexical token) is incorporated into the main verb in English. The third divergence type is a lexical divergence as illustrated in the stab example by the choice of a different lexical word dar (literally give) for the word $s t a b$. The fourth divergence type is categorial in that the predicate is adjectival (hungry) in English but nominal (hunger) in German. Finally, the fifth divergence type is a thematic divergence: the object (Mary) of the English sentence is translated as the subject (Maria) in the Spanish sentence.

The final divergence type, thematic divergence, is the one that will be the focus of this paper. We will look at 
how the UNITRAN system [Dorr, 1987, 1990] solves the thematic divergence problem by mapping an underlying lexical-conceptual structure to a syntactic structure (and vice versa) on the basis of a set of general linking routines and their associated mechanisms. The other divergences are also handled by the UNITRAN system, but these are discussed in [Dorr, 1990].

It turns out there are two types of thematic divergences that show up in the translation of a source language to a target language: the first type consists of a reordering of arguments for a given predicate; and the second type consists of a reordering of predicates with respect to their arguments or modifiers. We will look at examples of each of these types in turn.

In the first case, an example is the reversal of the subject with an object as in the English-Spanish example of gustar-like shown in figure 1. The predicate-argument structures are shown here: ${ }^{2}$

$$
\begin{aligned}
& \text { [I-Mex [N-MAX María] } \\
& \text { [V-MAX [V.1 [v.MIN me gusta] [P-MAX a mi] }]] \\
& \text { [I-MAX [N-MAX I] } \\
& \text { [V-MaX [v-1 [v-Mov like] [N-MaX Mary] }]]
\end{aligned}
$$

Here the subject Maria has reversed places with the object $m i$. The result is that the object mi turns into the subject $I$, and the subject Maria turns into the object Mary. The reverse wonld be true if translation went in the opposite direction.

An example of the second case of thematic divergence (not shown in figure 1) is the promotion of a complement up to the main verb, and the demotion of the main verb into an adjunct position (or vice versa). By promotion, we mean placement "higher up" in the syntactic structure, and by demotion, we mean placement "lower down" in the syntactic structure. This situation arises in the translation of the Spanish sentence Juan suele ir a casa into the English sentence John usually goes home:

(2)

$$
\begin{aligned}
& \text { [I-MAX [N-MAx Juan] } \\
& \text { [v-max [v-1 [v-min suele] } \\
& \mathbb{1} \\
& \text { [v-MAX [v.MaN ir] [P-MAX a casa]]]] } \\
& \text { [I-MAX [N-Mex John] } \\
& \text { [v.MaX [v.t [v-1 usually [v.Min goes]] } \\
& \text { [N-Max home]]]] }
\end{aligned}
$$

Here the main verb soler takes ir as a complement; but, in English, the ir predicate has been placed into a higher position as the main verb go, and soler is placed into a lower position as the adjunct usually associated with the main verb. The reverse would be true if translation went in the opposite direction.

\footnotetext{
${ }^{2}$ Often times a native speaker of Spanish will invert the subject to post-verbal position:

[I-MAX $e_{i}$ [v-Max [v-1 [v-MiN me gusta] [P-Max a mí]]] [N-Max Maria]i].

However, this does not affect the internal/external reversal scheme described here since inversion takes place independently after thematic divergences have been handled.
}

Another example of the second case of thematic divergence is the demotion of the main verb into a complement position, and the promotion of an adjunct up to the main verb (or vice versa). This situation arises in the translation of the German sentence Ich esse gern into the English sentence $I$ like eating:

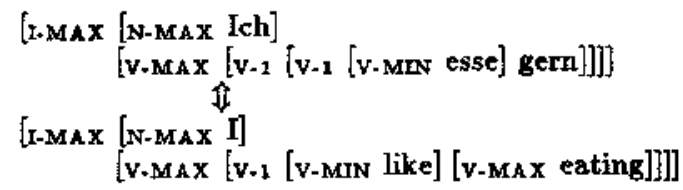

Here the main verb essen takes gern as an adjunct; but, in English, gern has been placed into a higher position as the main verb like, and the essen predicate has been placed into a lower position as the complement eating of the main verb. The reverse would be true if translation went in the opposite direction. ${ }^{3}$

This paper will show how the system uses three mechanisms along with a set of general linking routines (to be defined) to solve thematic divergences such as those that have been presented. The next section introduces the terminology and mechanisms that are used in the solution of these divergences, and, in so doing, it will provide a brief glimpse of how thematic divergences are tackled. Section 3 discusses other approaches (and their shortcomings) in light of the thematic divergence problem. Finally, section 4 presents a general solution for the problem of thematic divergences, showing in more detail how a set of general linking routines and their associated mechanisms provide the appropriate mapping from source to target language.

\section{TERMINOLOGY AND MECHANISMS}

Before we examine thematic divergences and how they are solved, we must first look at the terminology and mechanisns used throughout this paper:4

\footnotetext{
"It might be argued that a "direct" translation is possible for each of these three examples:

(1) Mary pleases me

(21) John is accustomed to going home

(31) I eat willingly

The problem with taking a direct approach is that it is not general enough to handle a wide range of cases. For example, gern can be used in conjunction with haben to mean like: Ich habe Marie gern ('I like Mary'). The literal translation, I have Mary likingly, is not only stylistically unattractive, but it is not a valid translation for this sentence. In addition, the direct-mapping approach is not bidirectional in the general case. Thus, even if we did take (1i), (2i), and (3i) to be the translations for (1), (2), and (3), we would not be able to apply the same direct mapping on the English sentences of (1), (2), and (3) (translating in the opposite direction) because we would still need to translate like and usually into Spanish and German. It is clear that we need some type of uniform method for translating thematic divergences.

${ }^{4}$ The terms complement, specifier, and adjunct have not been defined; roughly, these correspond to syntactic object,
} 
Definition 1: An $L C S$ is a lexical conceptual structure conforming to a modified version of Jackendoff's well-formedness rules [Jackendoff, 1983]. For example, I like Mary is represented as:

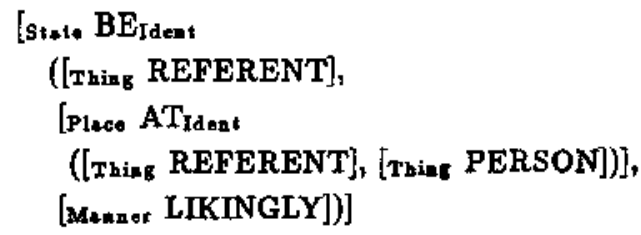

Definition 2: An RLCS is an uninstantiated LCS that is associated with a root word definition in the lexicon (i.e., an LCS with unfilled variable positions). For example, an RLCS associated with the word like is:

[siste $\mathbf{B E}_{\text {Tdent }}$

$$
\begin{aligned}
& ([\text { Thise X], } \\
& \text { [Place AT Adent }([\text { Thing X], [Thias Y])], } \\
& \text { [Menner LIKINGLY])] }
\end{aligned}
$$

Definition 3: A CLCS is a composed (instantiated) LCS that is the result of combining two or more RLCS's by means of unification (roughly). This is the interlingua or languageindependent form that is the pivot between the source and target language. For example if we compose the RLCS for like with the RLCS's for I ([Thirg REFERENT]) and Mary ([Thia PERSON]), we get the CLCS corresponding to I like Mary (as shown in definition 1).

Definition 4: An Internal Argument Position is a syntactic complement for a lexical word of category $\mathrm{V}, \mathrm{N}, \mathrm{A}, \mathrm{P}, \mathrm{I}$, or $\mathrm{C}^{5}$

Definition 5: An External Argument Position is a syntactic specifier of $\mathrm{N}$ for a lexical word of category $N$ or a specifier of $I$ for a lexical word of category $\mathrm{V}$.

Definition 6: An Adjunct Argument Position is a syntactic modifier that is neither internal nor external with respect to a lexical word.

Each word entry in the lexicon is associated with an RLCS, whose variable positions may have certain restrictions on them such as internal/external and promotion/demotion information (to be described). The CLCS is the structure that results from combining the lexical items of a source-language sentence into a single underlying pivot form.

subject, and modifier, respectively. For a more detailed description of these and some of the other definitions here, see [Dorr, 1990].

${ }^{\mathrm{V}} \mathrm{V}, \mathrm{N}, \mathrm{A}, \mathrm{P}, \mathrm{I}$, and C stand for Verb, Now, Adjective, Preposition, Inflection, and Complementizer, respectively.
The mapping that solves thematic divergences is defined in terms of the RLCS, the CLCS, the syntactic structure, and the markers that specify internal/external and promotion/demotion information. These markers, or mechanisms, are specified as follows:

Mechanism 1: The :INT and :EXT markers are override pasition markers that determine where the internal and external arguments will be positioned for a given lexical root word.

For example, the lexical entry for gustar is an RLCS that looks like the RLCS for like (see definition 2) except that it includes the :INT and :EXT markers:

[s1ate $B_{\text {Ident }}$

([Thing $X: I N T]$,

[Place $\mathrm{AT}_{\text {Idext }}([\mathrm{Thing}, \mathrm{X}]$, [Thing $\left.\left.\mathrm{Y}: \mathrm{EXT}]\right)\right]$,

[Mannor LIKINGLY])]

During the mapping from the CLCS (shown in definition 1) to the syntactic structure, the RLCS for gustar (or like) is matched against the CLCS, and the arguments are positioned according to the specification associated with the RLCS. ${ }^{6}$ Thus, the :INT and :EXT markers account for the syntactic distinction between Spanish and English by realizing the [rting REFERENT] node of the CLCS (corresponding to $X$ in the RLCS) as the internal argument $m i$ in Spanish, but as the external argument $I$ in English; and also by realizing the [Tring PERSON] node of the CLCS (corresponding to $\mathrm{Y}$ in the RLCS) as the external argument María in Spanish, but as the internal argument Mary in English. Note that the :INT and :EXT markers show up only in the RLCS. The CLCS does not include any such markers as it is intended to be a language-independent representation for the source and target-language sentence.

Mechanism 2: The :PROMOTE marker associated with an RLCS $\mathcal{H}$ places a restriction on the complement $\mathcal{P} /$ of the head $\mathcal{H} l^{7}$ This restriction forces $\mathcal{P} I$ to be promoted in the CLCS as the head $\mathcal{P}$. $\mathcal{H}$ is then dropped into a modifier position of the CLCS, and the logical subject of $\mathcal{P}$ is inherited from the CLCS associated with the syntactic subject of $\mathcal{H} 1^{8}$

For example, the lexical entry for soler contains a :PROMOTE marker that is associated with the RLCS: [Ma*ner HABITUALLY :PROMOTE]

Thus, in the above formula $\mathcal{H} /$ corresponds to soler, and $\mathcal{P} t$ corresponds to the complement of soler. The :PROMOTE marker forces the syntactic complement $\mathcal{P}$, to be promoted into head

\footnotetext{
${ }^{6}$ The lexical-selection procedure that maps the CLCS to the appropriate RLCS (for like or gustar) is not described in detail here (see [Dorr, 1990]). Roughly, lexical selection is a 129 unification-like process that matches the CLCS to the RLCS templates in the lexicon, and chooses the associated lexical words accordingly.
} 
position as $\mathcal{P}$ in the CLCS, and the head $\mathcal{H} /$ to be demoted into modifier position as $\mathcal{H}$ in the CLCS. So, in example (2) of the last section, the resulting CLCS is: ${ }^{9}$

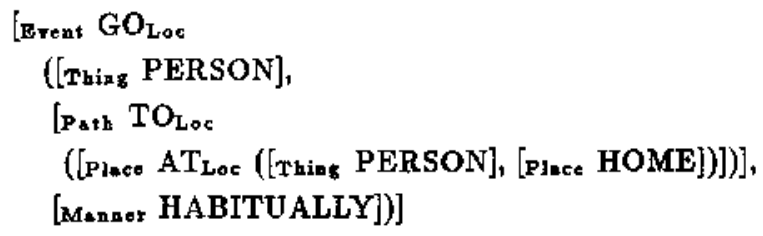

Here the RLCS for soler, [Moner HABITUALLY], corresponds to $\mathcal{H}$ and the RLCS for ir, [event GO ...], corresponds to $\mathcal{P}$. In the translation to English, [Manan HABITUALLY] is not promoted, so it is realized as an adjunct usually of the main verb go.

Mechanism 3: The :DEMOTE marker associated with an RLCS $\mathcal{P}$ places a restriction on the head $\mathcal{H}$ ' of the adjunct $\mathcal{P}$. This restriction forces $\mathcal{H}$ to be demoted into an argument position of the CLCS, and the logical subject of $\mathcal{P}$ to be inherited from the logical subject of $\mathcal{H}$.

For example, the lexical entry for gern contains a :DEMOTE marker that is associated with the $\mathrm{Y}$ argument in the RLCS:

[state BE Circ

([Thing $\mathrm{X}]$,

[Plece AT oire $_{\text {([thing } X]}$ ], [Eront $Y$ :DEMOTE])],

[Mosner LIKINGLY])]

Thus, in the above formula, $\mathcal{P}$ f corresponds to gern and $\mathcal{H} /$ corresponds to the syntactic head that takes gern as an adjunct. The :DEMOTE marker forces the head $\mathcal{H} I$ to be demoted into an argument position as $\mathcal{H}$ in the CLCS, and the adjunct $\mathcal{P} /$ to be promoted into head position as $\mathcal{P}$ in the CLCS. So in example (3) of the last section, the resulting CLCS is:

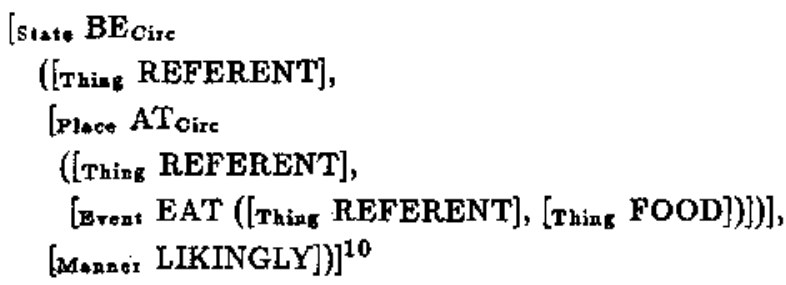

Here the RLCS for gern, [stal BE $\left.\mathrm{B}_{\text {Circ }} \ldots\right]$, corresponds to $\mathcal{P}$ and the RLCS for essen, [siate EAT ...], corresponds to $\mathcal{H}$. In the translation to English, [state $B E_{\text {Cire }} . .$. ] is not demoted, so it is realized as the main verb like that takes eating as its complement.

\footnotetext{
'In general, a syntactic argument $u$ ' is the canonical syntactic realization $(\mathcal{C S R})$ of the corresponding CLCS argument $u$. The $\mathcal{C S R}$ function is a modified version of a routine proposed in [Chomsky, 1986]. See [Dorr, I990] for a more detailed discussion of this function.

The logical subject is the highest/left-most argument in the CLCS.
}

Now that we have looked briefly at the mechanisms involved in solving thematic divergences in UNITRAN, we will look at how other approaches have attempted to solve this problem.

\section{PREVIOUS APPROACHES}

In tackling the more global problem of machine translation, many people have addressed different pieces of the thematic divergence problem, but no single approach has yet attempted to solve the entire space of thematic divergence possibilities. Furthermore, the pieces that have been solved are accounted for by mechanisms that are not general enough to carry over to other pieces of the problem, nor do they take advantage of cross-linguistic uniformities that can tie seemingly different languages together.

Gretchen Brown has provided a model of GermanEnglish translation that uses lexical semantic structures [Brown, 1974]. The work is related to the model developed for UNITRAN since both use a form of conceptual structure as the basis of translation. While this approach goes a long way toward solving a number of translation problems (especially compound noun disambiguation), it falls short of providing a systematic solution to the thematic divergence problem. This is largely because the conceptual structure does not serve as a common representation for the source and target languages. Instead, it is used as a point of transfer, and as spch, it is forced to encode certain language-specific idiosyncrasies such as the syntactic positioning of conceptual arguments. In terms of the representations used in UNITRAN, this approach is analogous to using a language-to-language mapping from the RLCS's of the source langusge to the RLCS's of the target language without using an intermediate language-independent structure as a pivot form. In

\footnotetext{
${ }^{9}$ It should be noted that promotion and demotion structures are inverses of each other. Thus, although this CLCS looks somewhat "English-like," it is possible to represent the CLCS as something that looks somewhat "Spanish-like:"

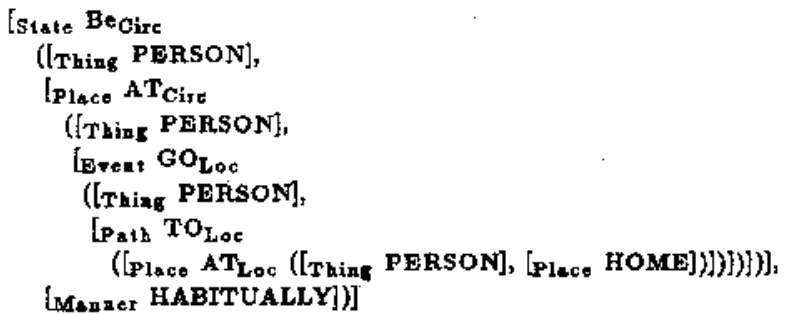

In this case, we would need to use the :DEMOTE marker (see mechanism 3) instead of the :PROMOTE marker, but this marker would be used in the RLCS associated with usually instead of the RLCS associated with soler. The justification for using the "English-like" version for this example is that the [Manaer HABITUALLY] constituent is generally thought of as an aspectual element associated with a predicate (e.g., in German, the sentence would be Ich gehe gewöhnlich nach Hause ('I go usually home')); this constituent cannot be used as a predicate in its own right. Thus, the complicated "Spanish-like" predicate-argument structure is not a likely conceptual representation for constructions that use [Manner HABITUALLY].

${ }^{10}$ The default object being eaten is [Thixg FOOD], although this is not syntactically realized in this example.
} 
this approach, there is no single language-independent mechanism that links the conceptual representation to the syntactic structure; thus, it is necessary to handcode the rules of thematic divergence for English and German, and all divergence generalizations are lost.

In 1982, Lytinen and Schank developed the MOPTRANS Spanish-English system based on conceptual dependency networks [Lytinen \& Schank, 1982]. ${ }^{11}$ This approach is related to the UNITRAN model of translation in that it uses an interlingual representation as the pivot from source to target language. The key distinction is that the approach lacks a generalized linking to syntax. For example, there is no systematic method for determining which conceptual argument is the subject and which is the object. This means that there is no uniform mechanism for handling divergences such as the subject-object reversal of example (1).

The LMT system is a logic-based English-German machine translator based on a modular logical grammar [McCord, 1989]. McCord specifically addresses the problem of thematic divergence in translating the sentence Mir gefallt der Wagen (I like the car). However, the solution that he offers is to provide a "transfer entry" that interchanges the subject and object positions. There are two problems with this approach. First it relies specifically on this object-initial ordering, even though the sentence is arguably more preferable with a subject-initial ordering Der Wagen gefallt mir; thus, the solution is dependent on syntactic ordering considerations, and will not work in the general case. Second the approach does not attempt to tie this particular type of thematic divergence to the rest of the space of thematic divergence possibilities; thus, it cannot uniformly translate a conceptually similar sentence Ich fahre das Wagen gern (I like to drive the car).

\section{THEMATIC DIVERGENCES}

In section 1 , we introduced some examples of thematic divergences, and in section 2 we described some of the mechanisms that are used to solve these divergences. Now that we have looked at other machine translation approaches with respect to the thematic divergence problem, we will look at the solution that is used in the UNITRAN system.

Recall that there are two types of thematic divergences:

1. Different argument positionings with respect to a given predicate.

2. Different predicate positionings with respect to arguments or modifiers.

The first type covers the case of argument positions that diverge; it is accounted for by the :INT and :EXT markers. The second type covers the case of predicate positions that diverge; it is accounted for by the :PROMOTE

\footnotetext{
${ }^{11}$ Several researchers have worked within this framework including Goldman [1974], Schank \& Abelson [1977], and many others.
}

and :DEMOTE markers. Together, these two types of divergences account for the entire space of thematic divergences, since all participants must be one of these two (either an argument, or a predicate, or both).

In both cases of thematic divergence, it is assumed that there is a CLCS that is derived from a sourcelanguage RLCS that is isomorphic to the correspond ing target-language RLCS (i.e., the variables in the 2 RLCS's map to the same positions, though they may be labeled differently). Furthermore, it is assumed that thematic divergence arises only in cases where there is a logical subject.

A CLCS with logical subject $w$, non-subject arguments $z_{1}, z_{2}, \ldots, z_{k}, \ldots, z_{n}, \quad$ and modifiers $n_{1}, n_{2}, \ldots, n_{l}, \ldots, n_{m}$ will look like the structure shown in (4), where the dominating head $\mathcal{P}$ is a typed primitive $\left(e . g \cdot, \mathrm{BE}_{\text {Circ }}\right)$ :

(4)

$$
\left[\mathcal{P} w, z_{1}, z_{2}, \ldots, z_{k}, \ldots, z_{n}, n_{1}, n_{2}, \ldots, n_{l}, \ldots, n_{m}\right]
$$

In order to derive the syntactic structure from the CLCS, we need a mapping or linking rule between the CLCS positions and the appropriate syntactic positions. Roughly, this linking rule is stated as follows:

General Linking Routine $\mathcal{G}$ :
(a) Map the logical subject to the external argu- ment position.
(b) Map the non-logical-subjects to internal ar- gument positions.
(c) Map modifiers to adjunct positions.
(d) Map the dominating head to the phrasal head position.

$\mathcal{G}$ is used for the second half of translation (i.e., mapping to the target-language structure); we also need an inverse routine that maps syntactic positions of the sourcelanguage structure to the CLCS positions:

Inverse Linking Routine $\mathcal{G}^{-1}$ :

(a) Map the external argument to the logical subject position.

(b) Map the internal arguments to non-logicalsubject positions.

(c) Map adjuncts to modifier positions.

(d) Map the phrasal head to the dominating head node.

In terms of the representation shown in (4), the $\mathcal{G}$ and $\mathcal{G}^{-1}$ mappings would be defined as shown in figure $2.12,13,14$ Note that $w t, z_{1} t, \ldots, z_{k} l, \ldots, z_{n} l$, and $n_{1} l, \ldots, n_{l} f, \ldots, n_{m}$ are the source-language realizations of the corresponding CLCS tokens $w$, $z_{1}, \ldots, z_{k}, \ldots, z_{n}$, and $n_{1}, \ldots, n_{l}, \ldots, n_{m} ;$ similarly, $w n$, $z_{1} \prime, \ldots, z_{k} n, \ldots, z_{n} \prime \prime$, and $n_{1} \prime, \ldots, n_{l} \prime, \ldots, n_{m} \prime \prime$ are target-language realizations of the same CLCS tokens. This assumes that there is only one external argument and zero or more internal arguments. We will now look 


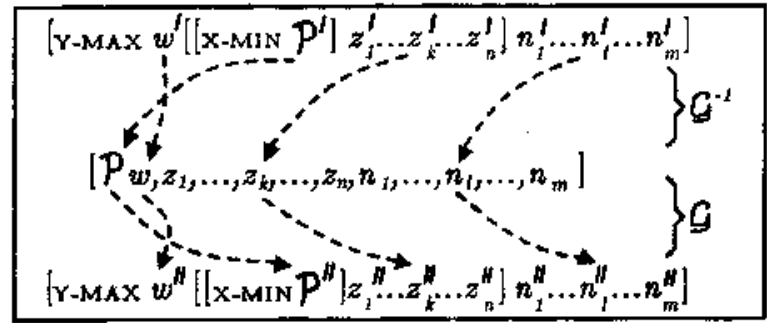

Figure 2: Mapping From Source to Target via the CLCS

at a formal description of how each type of thematic divergence is manifested. We will then see how the general linking routines described here take the syntactic mechanisms into account in order to derive the appropriate result.

\subsection{Divergent Argument Positionings}

In order to account for the thematic reversal that shows up in the gustar-like example of (1), we must have a mechanism for mapping CLCS arguments to different syntactic positions. In terms of the CLCS, we need to allow the syntactic realization of the logical subject $w$ and the syntactic realization of a non-subject argument (say $z_{k}$ ) to switch places between the source and target language.

Figure 3 shows how this type of argument reversal is achieved. The :INT and :EXT markers are used in the RLCS specifications as override markers for the $\mathcal{G}$ and $\mathcal{G}^{-1}$ routines: the :INT marker is used to map the logical subject of the CLCS to an internal syntactic position (and vice versa). Thus, steps (a) and (b) of $\mathcal{G}$ and $\mathcal{G}^{-1}$ are activated differently if the RLCS associated with the phrasal head contains either of the :INT or :EXT override mechanisms. Note that the CLCS is the same for

${ }^{12}$ The convention adopted in this paper is to use $u$ for the source-language realization, and $u \prime l$ for the target-language realization for a CLCS argument $u$.

${ }^{13}$ Adjunction has been placed to the right at the maximal level. However, this is not the general case. A parameter setting determines the side and level at which a particular adjunct will occur (as discussed in [Dorr, 1990]). The configuration shown corresponds to the spec-initial/headinitial case. The other three possible configurations are:

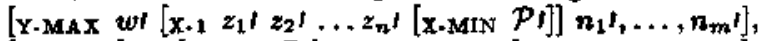

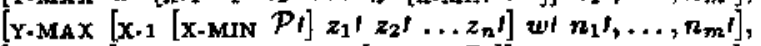

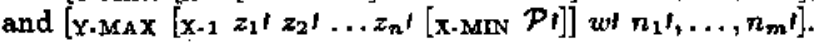

Finally, the order of the $z_{i}$ ' 's and $n_{j}$ 's is not being addressed here; this is determined by independent principles also discussed in [Dorr, 1990]. Regardless of these syntactic variations, the $\mathcal{G}$ and $\mathcal{G}^{-1}$ routines operate uniformly because they are language-independent. For simplicity, the specinitial/head-initial configuration will be used for the rest of this paper.

${ }^{14}$ In addition to realization of arguments, the dominating CLCS head $(\mathcal{P})$ must also be realized as a lexical word $(\mathcal{P}$, in the source language and $\mathcal{P} \|$ in the target language). The syntactic category of this lexical word is $X$, and the maximal projection is $\mathrm{Y}$-MAX. In general, $\mathrm{Y}=\mathrm{X}$ unless $\mathrm{X}$ is a Verb (in which case, $Y$ is the Inflection category).

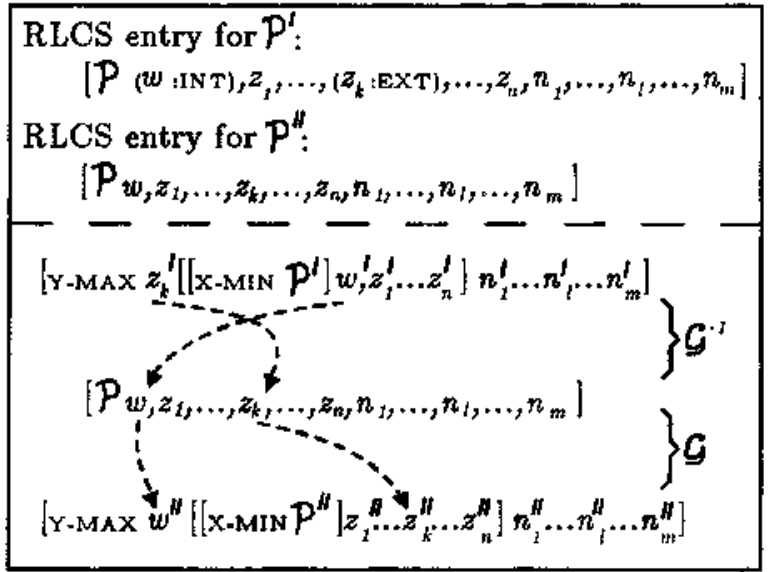

Figure 3: Mapping From Source to Target for Divergent Arguments

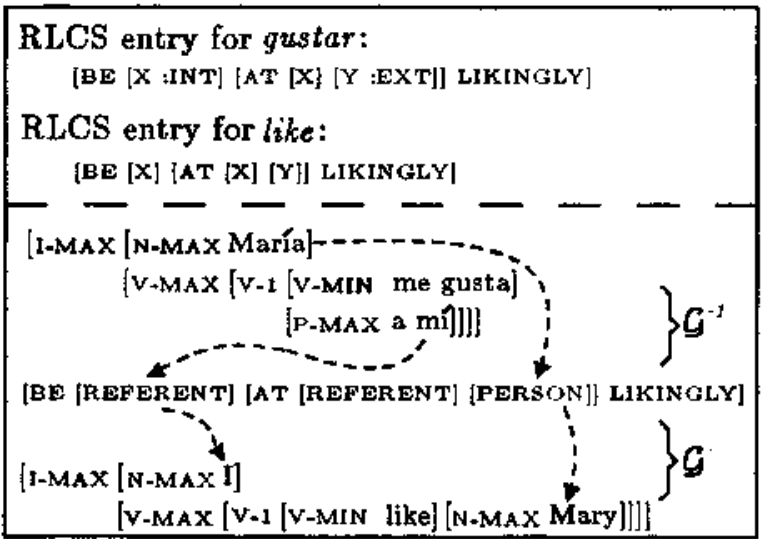

Figure 4: Translation of María me gusta a mí

both the source and target language; only the RLCS's in the lexical entries need to include language-specific information in order to account for thematic divergences. Now using the $\mathcal{G}$ and $\mathcal{G}^{-1}$ routines and the overriding :INT and :EXT mechanisms, we can show how to account for the thematic divergence of example (1).

Figure 4 shows the mapping from Spanish to English for example (1). ${ }^{15,16}$ Because the Spanish RLCS includes the :INT and :EXT markers, the $\mathcal{G}^{-1}$ routine activates steps (a) and (b) differently: the external argument Maria is mapped to a non-logical-subject position [Thing PERSON], and the internal argument mí is mapped to the logical subject position [Thing REFERENT]. By

\footnotetext{
${ }^{15}$ Because of space limitations, we will illustrate the three examples (1), (2), and (3) in one direction only. However, it should be clear that the thematic divergences are solved going in the opposite direction as well since the $\mathcal{G}$ and $\mathcal{G}^{-1}$ mappings are reversible.

${ }^{16}$ A shorthand notation is being used for the RLCS's and the CLCS. See section 2 for a description of the actual representations used by the system.
} 
contrast, the English RLCS does not include any special markers. Thus, the $\mathcal{G}$ routine activates steps (a) and (b) normally: the logical subject [Thing REFERENT] is mapped to the external argument $I$, and the nonlogical-subject [Thing PERSON] is mapped to the internal position Mary.

Now we have seen how argument positioning divergences are solved during the translation process. ${ }^{17}$ In the next section, we will look at how we account for the second part of thematic divergences: different predicate positionings.

\subsection{Divergent Predicate Positionings}

In the last section, we concentrated primarily on thematic interchange of arguments. In this section, we will concentrate on thematic interchange of predicates. In so doing, we will have accounted for the entire space of thematic divergences.

There are two ways to be in a predicate-argument relationship: the first is by complementation, and the second is by adjunction. That is, syntactic phrases include basegenerated complements and base-generated adjuncts, both of which participate in a predicate-argument structure (where the predicate is the head that subcategorizes for the base-generated complement or adjunct). ${ }^{18}$

In order to show how predicate divergences are solved, we must enumerate all possible sourcelanguage/target-language predicate positionings with respect to arguments $z_{1}, z_{2}, \ldots, z_{k}, \ldots, z_{n}$ and modifiers $n_{1}, n_{2}, \ldots, n_{l}, \ldots, n_{m}$. In terms of the syntactic structure, we must examine all the possible positionings for syntactic head $\mathcal{P}$, with respect to its complements $z_{1} t, z_{2} t, \ldots, z_{k} \prime, \ldots, z_{n} t$ and adjuncts $n_{1} t, n_{2} l, \ldots, n_{l} l, \ldots, n_{m} t$.

\footnotetext{
${ }^{17}$ It should be noted that the solution presented here (as well as that of the next section) does not appeal to an alreadycoded set of conceptual "frames." Rather, the syntactic siructures are derived procedurally on the basis of two pieces of information: lexical entries (i.e., the RLCS's) and the result of composing the RLCS's into a single unit (i.e., the CLCS). It would not be possible to map declaratively, i.e., from a set of static source-language frames to a set of static target-language frames. This is because the $\mathcal{G}$ and $\mathcal{G}^{-1}$ routines are intended to operate recursively: an argument that occurs in a divergent phrasal construction might itself be a divergent phrasal construction. For example, in the sentence le auele gustar leer a Juan ('John usually likes to read'), there is a simultaneous occurrence of two types of divergences: the verb soler exhibits a predicate positioning divergence with respect to its complement gustar leer a $J$ uan, which itself exhibits an argument positioning divergence. The procedural mappings described here are crucial for handling such cases.

${ }^{18}$ We have left out the possibility of a base-generated specifier as a participant in the predicate-argument relationship. Of course, the specifier is an argument to the predicate, but it turns out that the syntactic specifier, which corresponds to the logical subject in the LCS, has a special status, and does not participate in predicate divergences in the same way as syntactic complements and adjuncts. This will be illustrated shortly.
}

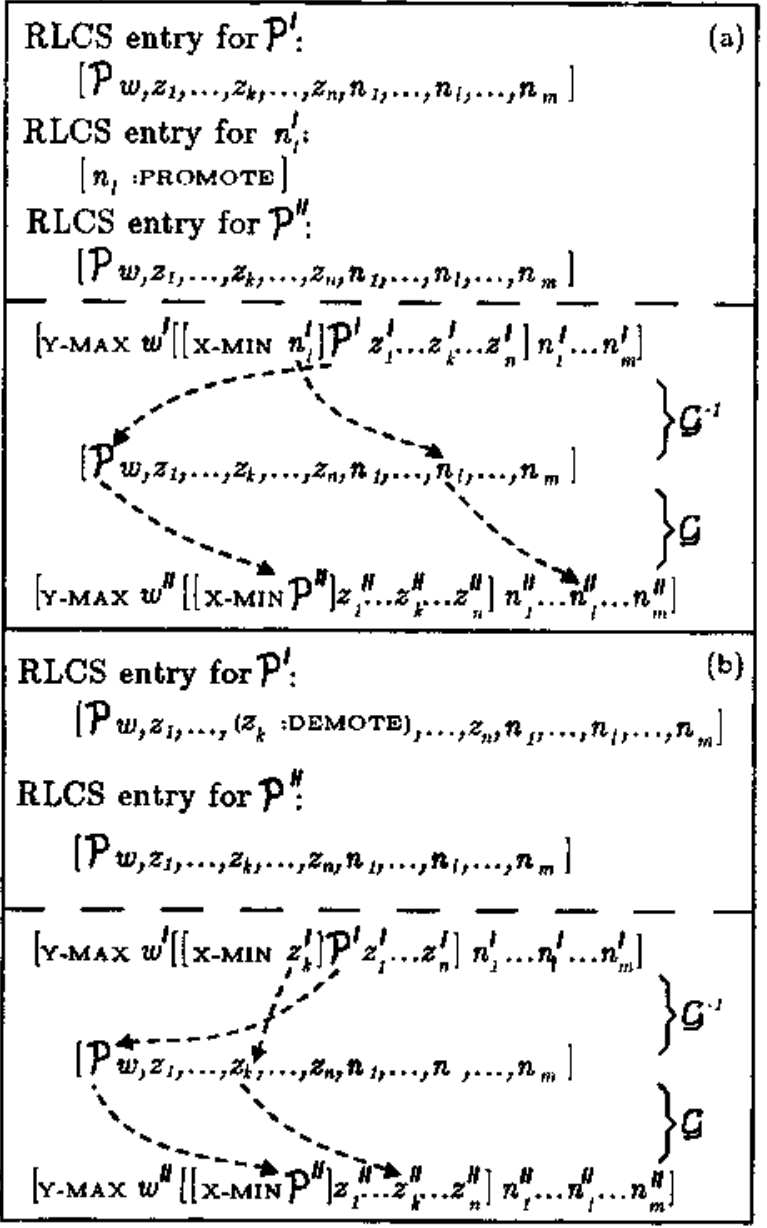

Figure 5: Mapping From Source to Target for Divergent Predicates

There are a large number of possible positionings that exhibit predicate divergences, but only two of them arise in natural language. ${ }^{10}$ It turns out that the solerusually example of (2) and the gern-like example of (3) are representative of the space of possibilities of predicate divergences. The source-language/target-language predicate positionings for these two cases are represented as shown in figure 5. Part (a) of this figure accounts for the translation of usually to soler (or vice versa), and part (b) accounts for the translation of like to gern (or vice versa).

The $\mathcal{G}$ and $\mathcal{G}^{-1}$ routines do not take into account the predicate divergences that were just presented. As in the case of argument divergences, predicate divergences require override markers. The :PROMOTE marker is used to map a modifier of the CLCS to a syntactic head position (and vice versa). The :DEMOTE marker is used to map a non-subject argument of the CLCS to a syntactic head position (and vice versa). Thus, steps (c), and

\footnotetext{
${ }^{19}$ There is not enough space to elaborate on this claim here. See [Dorr, 1990] for a detailed discussion of what the possible positionings are, and which ones make sense in the context of linguistic structure.
} 


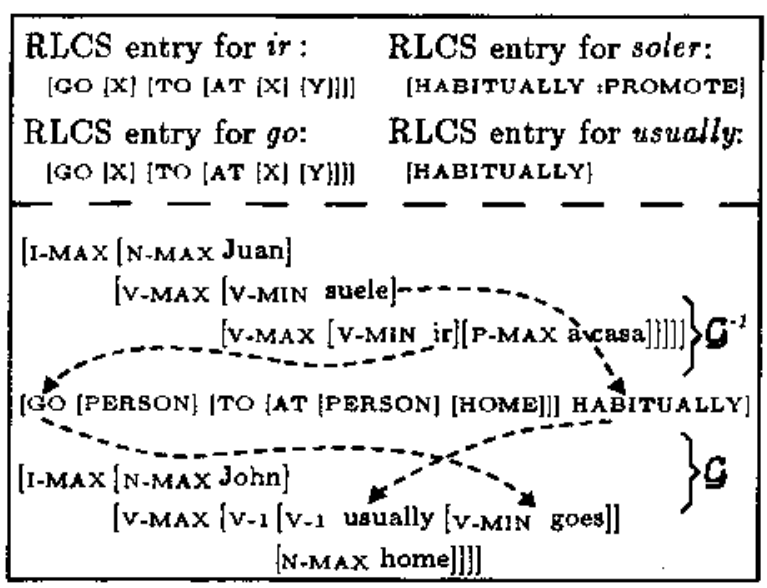

Figure 6: Translation of Juan suele ir a casa

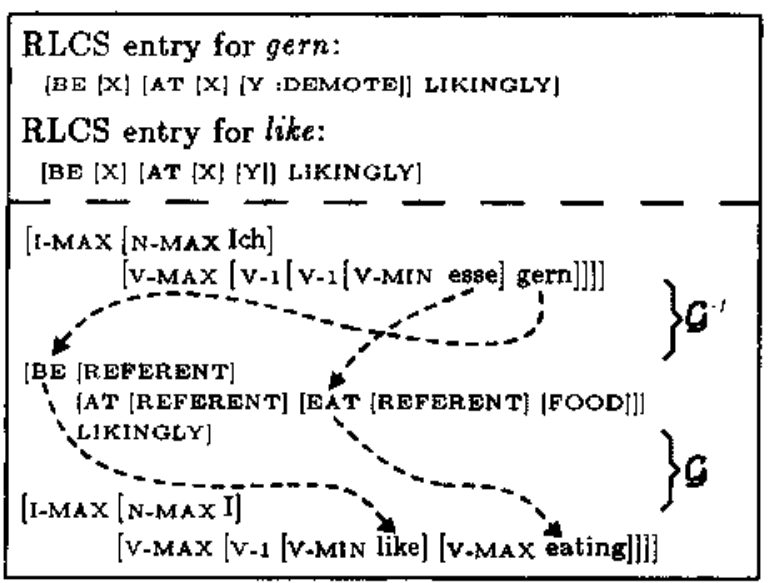

Figure 7: Translation of Ich habe Marie gern (d) of the $\mathcal{G}$ and $\mathcal{G}^{-1}$ routines are activated differently if the RLCS associated with the phrasal head contains the :PROMOTE override marker, and steps (b) and (d) of these routines are activated differently if a phrasal adjunct contains the :DEMOTE override marker.

Now using the $\mathcal{G}$ and $\mathcal{G}^{-1}$ routines and the overriding :PROMOTE and :DEMOTE mechanisms, we can show how to account for the thematic divergences of examples (2) and (3) (see figures 6 and 7, respectively).

In figure 6, the Spanish RLCS for soler includes the :PROMOTE marker. Thus, steps (c) and (d) of $\mathcal{G}^{-1}$ are overridden: the internal argument ir a casa is promoted into the dominating head position [erent $\mathrm{GO}_{\text {tocc }}$; and the phrasal head suele is mapped into a modifier position [Monaer HABITUALLY]. By contrast, the English RLCS does not include any special markers. Thus, the $\mathcal{G}$ routine activates steps (c) and (d) normally: the dominating head [Brest $\mathrm{GO}_{\text {Loc }}$ ] is mapped into the phrasal head goes; and the modifier [Manner HABITUALLY] is mapped into an adjunct position usually.

In figure 7, the German RLCS for gern includes the :DEMOTE marker (associated with the variable $\mathbf{Y}$ ). Thus, steps (b) and (d) of $\mathcal{G}^{-1}$ are overridden: the phrasal head esse is demoted into a non-logical-subject position [Event EAT]; and the adjunct gern is mapped into the dominating head position [siste $\mathrm{BE}_{\text {Circ }}$. By contrast, the English RLCS does not include any special markers. Thus, the $\mathcal{G}$ routine activates steps (b) and (d) normally: the dominating head [state $B E_{\text {Circ }}$ ] is mapped into the phrasal head like; and the non-logical-subject [Event EAT] is mapped into the internal position eating.

\section{SUMMARY}

This paper has presented a solution to the problem of thematic divergences in machine translation. The solution has been implemented in UNITRAN, a bidirectional system currently operating on Spanish, English, and German, running in Commonlisp on a Symbolics 3600 series machine. We have seen that the procedures involved are general enough to operate uniformly across different languages and divergence types. Furthermore, the entire space of thematic divergence possibilities is covered in this approach without recourse to languagespecific routines or transfer rules. In addition to thematic divergences, the system handles the other divergence types shown in figure 1 , and it is expected that additional divergence types will be handled by means of equally principled methods.

\section{REFERENCES}

[Brown, 1974] Gretchen Brown, "Some Problems in German to English Machine Translation," MAC Technical Report 142, Massachusetts Institute of Technology, Cambridge, MA, 1974.

[Chomsky, 1986] Noam A. Chomsky, Knowledge of Language: Its Nature, Origin and Use, MIT Press, Cambridge, MA, 1986.

[Dorr, 1987] Bonnie J. Dorr, "UNITRAN: A Principle-Based Approach to Machine Translation," AI Technical Report 1000, Master of Science thesis, Department Electrical Engineering and Computer Science, Massachusetts Institute of Technology, Cambridge, MA, 1987.

[Dorr, 1990] Bonnie J. Dorr, "Lexical Conceptual Structure and Machine Translation," Ph.D. thesis, Department of Electrical Engineering and Computer Science, Massachusetts Institute of Technology, Cambridge, MA, 1990.

[Goldman, 1974] Neil M. Goldxnan, "Computer Generation of Natural Language from a Deep Conceptual Base," Ph.D thesis, Computer Science Department, Stanford University, Stanford, CA, 1974.

[Jackendoff, 1983] Ray S. Jackendoff, Semantics and Cognition, MIT Press, Cambridge, MA, 1983.

[Lytinen \& Schank, 1982] Steven Lytinen and Roger Schank, "Representation and Translation," Technical Report 234, Department of Computer Science, Yale University, New Haven, CT, 1982.

[McCord, 1989] Michael C. McCord, "Design of LMT: A Prolog-Based Machine Translation System," Computational Linguistics, 15:1, 33-52, 1989.

[Schank \& Abelson, 1977] Roger C. Schank and Robert Abelson, Scripts, Plans, Goals, and Understanding, Lawrence Er]baum Associates, Inc., Hillsdale, N J, 1977. 